\title{
INVESTIGATION OF RADIATION TRAPPING IN ATOMIC VAPOUR EXCITED BY WEAK RESONANT LASER PULSES
}

\author{
J. CHORAŻ̇Y AND T. STACEWICZ \\ Institute of Experimental Physics, Warsaw University \\ Hoża 69, 00-681 Warsaw, Poland
}

(Received July 6, 1999)

\begin{abstract}
We present results of onr investigations on radiation trapping in sodium vapour excited to $3 P$ level by weak laser pulses. The evolution of the fluorescence signal as well as the evolution of excited atoms distribution were studied using the Monte Carlo method. Some predictions of these investigations were checked experimentally.
\end{abstract}

PACS numbers: $42.50 . \mathrm{Hz}, 42.50 . \mathrm{Md}, 42.88 .+\mathrm{h}$

\section{Introduction}

The radiation imprisonment, which is also called the light diffusion, consists on multiple reemission and reabsorption of the resonance photons in optically dense medium. Macroscopically the light trapping usually leads to lengthening of the measured decay time of the resonance fiuorescence in comparison to the decay time observed for the optically thin medium.

Due to development in optics and in the computer techniques a fast progress in understanding of the radiation trapping mechanisms has been observed recently [1]. A good comprehending of the radiation trapping is important not only to understand the fluorescence phenomena. The radiation imprisonment affects the excited atoms distribution as well. The linowledge of such distribution is important for atomic spectroscopy, photochemistry, plasma physics, astrophysics, etc. The light diffusion should be also taken into account for explanation of phenomena in dense laser media, atomic line filters, fluorescent lamps as well as in objects recently generated due to laser cooling, like optical molasses and Bose-Einstein condensates $[2,3]$. "Trapping appear's to be important in most experimental setups and in most engineering applications whese atomic vapours are involved" [1].

The first description of the radiation diffusion was undertaken by Compton [4] and then by Milne [5]. However, they tried to present this process as an 
ordinary diffusion of particles. The more correct model was presented by Biberman [6] and Holstein [7, 8]. Their theory is based on a linear rate equation for population of excited atoms number density $n_{2}(r, t)$

$$
\begin{aligned}
& \frac{\partial n_{2}(r, t)}{\partial t}=-A_{21} n_{2}(r, t) \\
& \quad+\int \frac{A_{21} n_{2}\left(r^{\prime}, t\right)}{4 \pi\left|r-r^{\prime}\right|^{2}} \int k(\nu) P(\nu) \exp \left[-\left|r-r^{\prime}\right| k(\nu)\right] \mathrm{d} \nu \mathrm{d}^{3} r^{\prime}
\end{aligned}
$$

where $A_{21}$ denotes the spontaneous emission coefficient, while $k(\nu)$ is the absorption coefficient and $P(\nu)$ - the emission line profile. The second part of the equation describes excitation of atoms in the investigated point $r$ due to emission of photons from all other points $r^{\prime}$. The solution of the equation was found as a multiexponential function

$$
f(t)=\sum_{i} a_{i} \exp \left(-\beta_{i} t\right)
$$

The values of the rates constants $\beta$ can be found using different approaches [8-11]. Usually the decay constant $\beta_{1}$ of the main mode $(i=1)$ is the biggest one and it is called the radiation diffusion rate. The correctness of this description was confirmed experimentally by several groups [12-15]. Further theories of the radiation trapping [16-23] were usually based on the Holstein-Bibeman approach.

In laser irradiated media the light diffusion can take place in a slightly different way. Due to the strong excitation, the saturation of the resonance transition can occur. Such a case has been investigated intensively within the last decade by several authors using numerical [24], a.nalytic [25-27] and experimental methods [27-29]. For the strong excitation the Holstein-Biberman equation should be modified. In opposite to the weak excitation where the absorption coefficient is proportional to absolute concentration of atoms $N$, for the strong excitation, the absorption coefficient is proportional to the difference of population between the ground and excited atomic states

$$
k \propto \frac{g_{2}}{g_{1}}\left[n_{2}(r, t)-n_{1}(r, t)\right]
$$

Due to this modification the absorption coefficient should be considered as a space and time dependent function. Therefore $\mathrm{Eq}$. (1) becomes nonlinear.

The strong excitation leads to new optical phenomena. It was stated [24] that for the pulsed excitation the concentration of excited atoms can decrease much faster than for the weak excitation because the saturation of the optical transition leads to the transparency of the medium for the resonant photons. It causes also a faster evolution of the fluorescence signal than that for the weak excitation. It is due to the fact that the fluorescence decreases not only due to the decay of the excited atoms concentration but also due to the evolution of reabsorption in the medium. The reabsorption, that is negligible just after the excitation, rises then because of the decrease in the excited atoms concentration. Due to interference of both effects, for some geometries (for example: strong excitation of the full optical medium), the fluorescence signal can decrease faster than the natural decay. This phenomenon was confirmed experimentally [27-29]. It can occur only within a 
short time after the excitation. When the strong excitation vanishes, the radiation diffusion becomes linear and it can be described using the ordinary equation of Holstein and Biberman. At longer times the decay of excited atoms concentration and the fluorescence signal can be approached by a single exponential function with the $\beta_{j}$ constrant.

The weak and strong excitations of the optical medium denote two extreme experimental situations. The weak excitation is usually relatively easy to realize in an experiment since it can be reached even by means of conventional methods like heating, electric discharge, or irradiation by weak sources of light, etc. The strong excitation leading to the full optical bleaching of the medium can be produced only by means of powerful pulsed lasers. However, in a real experiment quite often there is an intermediate situation: the exciting laser pulse is relatively weak and due to absorption of laser pulses their intensity and their spectrum vary along the path in the medium, so the excitation is not uniform. In this case the excitation can be strong in the region near the entrance of the laser beam to the medium, however, it decreases toward the regions situated deeper. Then the fluorescence should depend on the position of the light detector in respect of the cell.

\section{Experiment}

The imprisomment of resonance radiation was investigated in sodium vapour excited to $3 S_{1 / 2} \rightarrow 3 P_{1 / 2}$ transition. The experimental setup is presented in Fig. 1 . The sodium was contained in a cell made of pyrex glass. The internal diameter of the cell was $2 \mathrm{~cm}$ while its length was about $4 \mathrm{~cm}$. The glass was protected against the aggressive influence of sodium by a borax layer [30]. In order to reduce the diffusion of the excited atoms to the walls, about 10 torr of neon was added as a buffer gas. The cell was placed in the oven, whose temperature was stabilized to $170^{\circ} \mathrm{C}$, corresponding to the sodium concentration of $10^{12} \mathrm{~cm}^{-3}[31]$. At such a low pressure the collisional broadening and the resonance broadening of the atomic line can be neglectied.

The sodium atoms were excited by $589.6 \mathrm{~nm}$ pulses from a dye laser pumped by second harmonic pulses of a Nd:YAG laser. The FWHM duration time of the

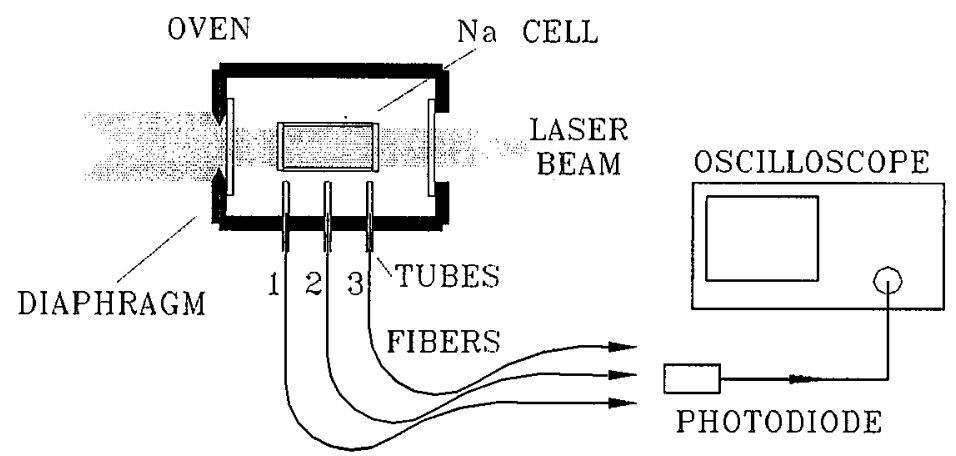

Fig. 1. The experimental setup. 
dye laser pulse was about $7 \mathrm{~ns}$. In order to assure a weak excitation of the vapour the laser pulse energy was limited to about $20 \mu \mathrm{J}$. The spectral line width of the laser light was about $30 \mathrm{GHz}$. One can evaluate that within the Doppler width of the resonance line $(2.4 \mathrm{GHz})$ the number of photons in the light pulse was smaller than the number of sodium atoms in the cell. Then, the laser pulse was able to excite strongly only the vapour near the entrance window. The excitation decreased along the distance from this window and at the end of the cell it was very weak. The full irradiation of the cell across the entrance window was assured by a careful shaping of the laser beam by the diaphragm.

A fluorescence signal was observed in the direction perpendicular to the laser beam. The light was collected by a set of optical fibers. In order to keep their position perpendicularly to the axis of the cell and to reduce their solid angle of observation, the fibers were placed in metal tubes. The tubes were situated in different positions in respect of the entrance window. During the experiment outputs of respective fibers were switched to the window of a photodiode. In this way the fluorescence could be observed from different regions of the cell. The rise time of the photodiode was about 0.3 ns. The photodiode signal was monitored by a $600 \mathrm{MHz}$ digital oscilloscope of $2 \mathrm{GHz}$ sampling rate (Hewlett Packard model HP 54600). In order to register very weak photon fluxes the photodiode signal was additionally amplified by an amplifier of $2 \mathrm{GHz}$ band pass.

The fluorescence signal registered near the entrance window is presented in Fig. 2. Here the laser pulse is strong enough to saturate the optical transition. The laser pulse starts at the zero point on the time scale and it causes the rise of the fluorescence. About 12 ns later, when the laser pulse is practically over, one reaches a maximum of the fluorescence and then the signal starts to decrease. Note that initially, for about 10 ns the decrease is faster than the natural decay. This is
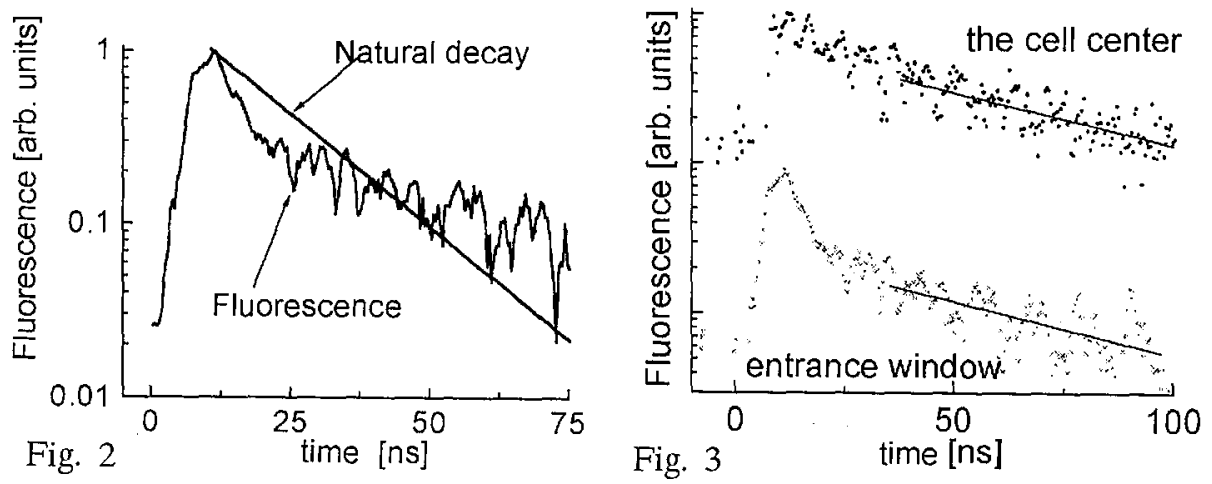

Fig. 2. Fluorescence signal registered near the entrance window of the sodium cell compared with the natural exponential decay of the $\mathrm{Na}(3 P)$ atoms $(\tau=16.7 \mathrm{~ns})$.

Fig. 3. Comparison of normalized fluorescence signal registered near the entrance window (strong excitation) with the fluorescence observed at the region situated about $2 \mathrm{~cm}$ apart (weak excitation). Continuous lines show the single mode decay characterized by a. constant of $110 \mathrm{~ns}$. 
a manifestation of the radiation imprisomment in the optically bleached medium, which was mentioned above. For the times longer than $25 \mathrm{~ns}$, one observes a slower decrease in the fluorescence signal with the time constant longer than the natural one. According to the model of Bezuglov et al. [26], when the fluorescence intensity decreases about 2-3 times in respect of its maximal value, the excitation is weak enough so that the radiation imprisonment can be described by the linear theory of Biberman and Holstein (1).

The fluorescence signals registered near the entrance window and the signal observed from the region about $2 \mathrm{~cm}$ apart (i.e. at the center of the cell) are compared in Fig. 3. The amplitudes of both signals are normalized to the unity. The signal registered far from the entrance window decreases much slower and no subnatural decay is observed. At times longer than $30 \mathrm{~ns}$ in both cases the single exponential function describes the fluorescence decrease. For the strong and weak excitations the decay time of the main mode of diffusion is practically the same and it reaches about $110 \pm 16 \mathrm{~ns}$.

\section{Monte Carlo model}

The radiation imprisonment which was investigated in the above experiment cannot be treated by means of numerical solution of integro-differential equation (1) modified for the optical bleaching. The initial distribution of the excited atoms is so nonuniform and asymmetric that such a nonlinear problem cannot be integrated even using the most powerful computers. Therefore we used the Monte Carlo modeling. As it has been already shown by Wiórkowski et al. [32, 33], Falęcki et al. [34-36], and Molish et al. [9-11], this technique can be successfully applied for solution of the radiation trapping problems.

In our model the experimental cell was divided in 40000 cube pixels whose edges $l$ were about $0.06 \mathrm{~mm}$ long. One assumes a homogeneous distribution of the excited atoms inside each pixel. Then the absorption coefficient, as well as the probability of the photon emission inside each pixel were found according to the local concentration of the ground state and excited atoms, respectively. A photon emitted from each pixel passed through one of 26 surrounding pixels. Using the Monte Carlo method the probability of its absorption in the surrounding pixel was determined. As a temporal step $\Delta t$ of iteration an interval of time corresponding to the time of propagation of light through the pixel was chosen $(\Delta t=l / c)$.

For sodium atom concentration of $10^{12} \mathrm{~cm}^{-3}$ in the presence of the buffer gas one can consider a Gaussian shape of the resonance line $(\lambda=589.6 \mathrm{~nm})$. In order to take into account the hyperfine splitting of the ground level $(1.7 \mathrm{GHz})$ the Doppler line width was corrected over the factor of 1.5 using the approximation of Colbert and Huennekens [15]. Finally, the atomic line width was estimated to be about $2.4 \mathrm{GHz}$. According to the experimental data we considered the spectral line width of the laser pulse equal to $0.015 \mathrm{~mm}$. A Gaussian temporal shape characterized by 7 ns FWHM duration time was assumed for the laser pulse. As usually we assume a full frequency redistribution in the vapour which means that the absorption and the emission spectra are the same.

The evolution of the excited atoms concentration is presented in Fig. 4. At the entrance window where the excitation is strong the saturation is achieved 

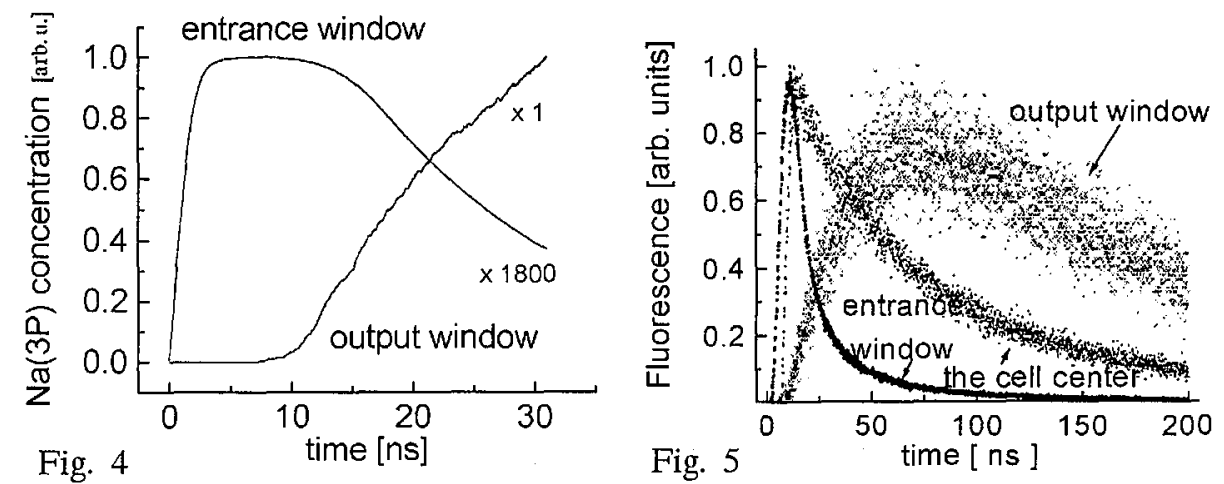

Fig. 4. Calculated evolution of concentration of the excited atoms at the axis of the cell at the entrance window and at the output window.

Fig. 5. Calculated fluorescence signals observed at the entrance window, at the cell center, and at the ontput window. Spread of the points manifests a statistical character of the model.

within 3 ns after beginning of the laser pulse and it remains high practically within the duration time of the laser pulse $(\approx 15 \mathrm{~ns})$. Then the concentration of excited a.toms decreases, however, clue to the optical bleaching this decrease is similar to the natural decay. A similar behavior of the $\mathrm{Na}(3 P)$ atoms was already modeled by means of other approaches [24-26], and was also confirmed experimentally [28, 29]. The evolution of concentration of the excited atoms looks differently at the end of the cell, where the excitation is negligible. The $n_{2}$ starts to rise practically about 10 ns after begimning of the laser pulse and it reaches the maximum approximately at about 80 ns. It shows that, when the laser pulse is weak enough to be absorbed in the medium, the excitation of atoms located farther in respect of the entrance window takes place mainly due to the light diffusion from the regions excited stronger, i.e. the regions located near the entrance window.

The results of calculations of the fluorescence signals for different regions of the sodium cell are presented in Fig. 5. The fluorescence registered near the entrance window decreases very fast and within several nanoseconds after the excitation its decay is even faster than the natural decay of the $3 P$ level. Here the shape of the signal is very similar to that presented in Fig. 3 . The statistical model confirms again the effect predicted by the numerical and analytic models [24-26], and the observations made earlier [27]. At the center of the cell, the excitation is slightly weaker, because the laser pulse intensity decreases due to the absorption in the cell. One considers that practically there is no saturation of the medium; the fluorescence signal decays apparently slower than the natural lifetime. Finally, at the output window the excitation is so small that one observes only the effect of the linear light diffusion: the rise of the signal takes place within about $80 \mathrm{~ns}$, i.e. much longer that the duration time of the laser pulse. The reason is that this region is irradiated by the resonance light from the other regions which are much stronger excited (situated closer to the entrance window). These results fit well the evolution of the concentration of excited atoms presented in Fig. 4. 


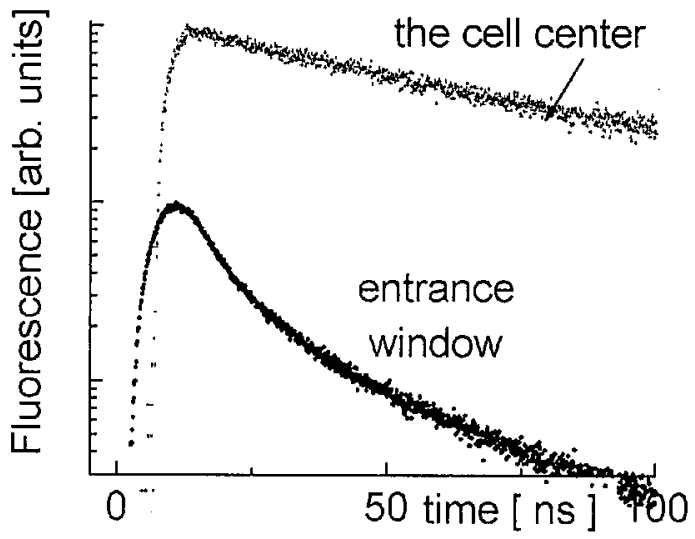

Fig. 6. Results of calculations of fluorescence signals at the entrance window and at the cell center.

The results of calculations of the fluorescence signals are presented in semilogarithmic scale in Fig. 6. One can see that the shape of the signals for the weak excitation (the cell center) corresponds well to the results of measurements presented in Fig. 3. For times longer than 30 ns the signal can be described by a single exponential function wilh the decay time of $100 \mathrm{~ns}$. That fits well the decay value found experimentally. As far as the strong excitation is concerned the calculated decrease in the signal is faster than that observed in the experiment. This discrepancy is mainly caused by relatively broad angle of observation of the fluorescence with the fiber (about $20^{\circ}$ ). It calused that in our experiment the observed light was coming also from the regions where the saturation was not so strong as it was assumed in the model.

\section{Conclusions}

Using experimental observations and the Monte Carlo model we investigated the light diffusion in sodium vapour excited by weak resonant laser pulses. At the enter of the cell, where the laser beam was strong enough to saturate the optical transition, one observes a fast decrease in the fluorescence signal that, within several nanoseconds after the excitation, decreases faster than the natural decay of $\mathrm{Na}(3 \mathrm{P})$ atoms. In the regions situated deeper in respect of the entrance window there is a weaker excitation that can be described by the linear theory of Holstein and Biberman. In the region located far from the input window the laser pulses are so weak that the excitation of the vapour takes place mainly due to the light diffusion from the regions excited stronger.

\section{References}

[1] A.F. Molish, B.P. Oehry, Radiation Trapping in Atomic Vapours, Oxford University Press, Oxford 1998.

[2] T. Walker, D. Sesko, C. Wieman, Phys. Rev. Lett. 64, 408 (1990).

[3] D.W. Sesko, T.G. Walker, C.E. Wieman, J. Opt. Soc. Am. B 8, 946 (1991).

[4] K.T. Compton, Phys. Rev. 20, 283 (1922). 
[5] E. Milne, J. Lond. Math. Soc. 1, 1 (1926).

[6] L.M. Biberman, Zh. Eksp. Teor. Fiz. 17, 416 (1947).

[7] T. Holstein, Phys. Rev. 72, 1212 (1947).

[8] T. Holstein, Phys. Rev. 83, 1159 (1951).

[9] A.F. Molish, B.P. Oehry, W. Schupita, G. Magerl, Comput. Phys. Commun. 74, 81 (1993).

[10] A.F. Molish, B.P. Oehry, W. Schupita, G. Magerl, Comput. Phys. Commun. 77, 255 (1993).

[11] A.F. Molish, B.P. Oehry, W. Schupita, G. Magerl, Comput. Phys. Commun. 93, 127 (1996).

[12] B.P. Kibble, G. Copley, L. Krause, Phys. Rev. 153, 153 (1967).

[13] J. Huennekens, A. Gallagher, Phys. Rev. A 28, 238 (1983).

[14] J. Huennekens, H.J. Park, T. Colbert, S.C. McClain, Phys. Rev. A 33, 2892 (1987).

[15] T. Colbert, J. Huennekens, Phys. Rev. A 41, 6154 (1990).

[16] C. van Trigt, Phys. Rev. 181, 97 (1969); Phys. Rev. A 1, 1298 (1970); Phys. Rev. A 4, 1303 (1971); Phys. Rev. A 13, 726 (1976); Phys. Rev. A 13, 734 (1976).

[17] M.G. Payne, J.D. Cook, Phys. Rev. A 2, 1238 (1970).

[18] M.G. Payne, J.E. Talmage, G.S. Hurst, E.B. Wagner, Phys. Rev. A 9, 1050 (1974).

[19] N. Bezuglov, A.N. Klyucharev, J. Quant. Spectrosc. Radiat. Transf. 34, 1 (1983).

[20] N. Bezuglov, W. Gorshkov, Opt. Spektrosk. 56, 1000 (1984).

[21] N. Bezuglov, Opt. Spektrosk. 58, 978 (1985).

[22] A.F. Molish, B.P. Oehry, W. Schupita, G. Magerl, J. Quant. Spectrosc. Radiat. Transf. 48, 377 (1992).

[23] A.F. Molish, B.P. Oehry, W. Schupita, G. Magerl, J. Quant. Spectrosc. Radiat. Transf. 49, 361 (1993).

[24] T. Stacewicz, T. Kotowski, P. Wiewiór, J. Chorąży, Opt. Commun. 100, 99 (1993).

[25] N. Bezuglov, A. Klucharev, B. Taratin, T. Stacewicz, A. Molish, F. Fuso, M. Allegrini, Opt. Commun. 120, 249 (1995).

[26] N.N. Bezuglov, A.N. Klucharev, A.F. Molisch, M. Allegrini, F. Fuso, T. Stacewicz, Phys. Rev. E 55, 3333 (1997).

[27] J. Chorązy, T. Kotowski, T. Stacewicz, Opt. Commun. 125, 65 (1996).

[28] G. Ankerhold, M. Schiffer, D. Mutschall, T. Sholtz, W. Lange, Phys. Rev. A 48, 4031 (1993).

[29] T. Scholtz, M. Schiffer, J. Welzel, D. Cysarz, W. Lange, Phys. Rev A 53, 2169 (1996).

[30] T. Stacewicz, J. Krasiński, J. Phys. E 16, 1028 (1983).

[31] N. Nesmeyanov, Vapour Pressure of Elements, Academic Press, New York 1963.

[32] P. Wiórkowski, W. Hartmann, Opt. Commun. 53, 217 (1985).

[33] P. Wiórkowski, Z. Phys. D 10, 417 (1988).

[34] W. Falęcki, W. Hartmann, P. Wiórkowski, Z. Phys. D 14, 111 (1988).

[35] W. Falęcki, W. Hartmann, R. Bocksch, Opt. Commun. 83, 215 (1991).

[36] M. Ostertag, W. Falęcki, W. Hartmann, Z. Phys. D 22, 383 (1991). 